\title{
A new class of weakly countably determined Banach spaces
}

by

\author{
K. K. Kampoukos and S. K. Mercourakis (Athens)
}

\begin{abstract}
A class of Banach spaces, countably determined in their weak topology (hence, WCD spaces) is defined and studied; we call them strongly weakly countably determined (SWCD) Banach spaces. The main results are the following: (i) A separable Banach space not containing $\ell^{1}(\mathbb{N})$ is SWCD if and only if it has separable dual; thus in particular, not every separable Banach space is SWCD. (ii) If $K$ is a compact space, then the space $C(K)$ is SWCD if and only if $K$ is countable.
\end{abstract}

1. Introduction. The aim of the present paper is to introduce and study a new class of Banach spaces referred to as strongly weakly countably determined (SWCD) spaces. This is, to some extent, a continuation of M-S], where strongly weakly $\mathcal{K}$-analytic (SWKA) Banach spaces were studied. Both classes generalize the corresponding one of strongly weakly compactly generated (SWCG) Banach spaces, introduced by G. Schlüchterman and R. F. Wheeler ([S-W]) and also presented in [H\&al]. The above classes are distinct and subclasses of the well known and extensively investigated classes WCD, WKA and WCG respectively. A significant point concerning the above mentioned subclasses is that none of them includes all separable Banach spaces and their members have interesting geometrical properties.

In Section 3, we strengthen the well known countably determined property for topological spaces by introducing the concept of a strongly countably determined (SCD) topological space (Def. 3.1). This concept generalizes the corresponding one of a strongly $\mathcal{K}$-analytic topological space, defined in M-S. We give several characterizations of strongly countably determined (and strongly $\mathcal{K}$-analytic) topological spaces, which are analogous to corresponding characterizations of countably determined (and $\mathcal{K}$-analytic) topological spaces (Props. 3.1 and 3.2 and also prove some stability properties of these spaces (Prop. 3.3). We show by Example 3.1 that a countably de-

2010 Mathematics Subject Classification: Primary 46B20, 54H05, 03E15; Secondary 46B26. Key words and phrases: WCD, SWCD Banach spaces, countably determined space, strongly countably determined space. 
termined (even $\mathcal{K}$-analytic) topological space need not be SCD. Finally we characterize SCD spaces as those topological spaces $X$ such that the space $\mathcal{K}(X)$ of compact nonempty subsets of $X$ endowed with the Vietoris topology is (strongly) countably determined (Th. 3.1). This result generalizes Th. 1.12 of $[\mathrm{M}-\mathrm{S}]$.

In Section 4, we define SWCD Banach spaces as those Banach spaces which are strongly countably determined in their weak topology (Def. 4.1) and study some of their properties. We give a characterization of SWCD Banach spaces using the lattice $\mathcal{K}(M)$ of compact nonempty subsets of a separable metric space $M$ (Prop. 4.1) in the spirit of similar characterizations of WKA ([T]), WCD ([M1], [A-M], C-O ]) and SWKA ([M-S] ) Banach spaces. We also prove that a separable Banach space $X$ not containing $\ell^{1}(\mathbb{N})$ is SWCD if and only if $X^{*}$ is separable (Th. 4.1); this result generalizes Th. 2.6 of [M-S]. It follows in particular that not every separable Banach space (even not containing $\ell^{1}(\mathbb{N})$ ) is SWCD. We also note that the classes of SWKA and SWCD Banach spaces are distinct, since according to a result of [M-S], a Banach space $X$ with separable dual is SWKA if and only if $X$ is Polish.

In Section 5, we study direct sums of SWCD Banach spaces. We show in particular that a countable $\ell^{p}$-sum (with $p \geq 1$ ) of SWCD (resp. SWKA) Banach spaces is also SWCD (resp. SWKA) (Prop. 5.1). On the other hand, in contrast to the case of WKA, WCD and WCG Banach spaces, we show that an uncountable $\ell^{p}$-sum $(p>1)$ of (separable) SWCD Banach spaces need not be SWCD, and an arbitrary uncountable $c_{0}$-sum of nontrivial Banach spaces is never SWCD (Th. 5.1 and Cor. 5.1). In particular $c_{0}\left(\omega_{1}\right)$ is not SWCD. As a consequence we prove that if $K$ is a compact Hausdorff space, then $C(K)$ is SWCD if and only if $K$ is countable (Th. 5.2).

2. Preliminaries and notation. We denote by $\Sigma$ the set $\mathbb{N}^{\mathbb{N}}$ of infinite sequences of positive integers, endowed with the cartesian topology, which makes $\Sigma$ (usually called the Baire space) a Polish space (i.e., homeomorphic to a complete separable metric space). $S$ stands for the set $\bigcup_{n=0}^{\infty} \mathbb{N}^{n}\left(\mathbb{N}^{0}=\emptyset\right)$ of finite sequences of positive integers. We give $S$ the partial order of "initial segments" which makes $S$ a tree: for $s=\left(s_{1}, \ldots, s_{n}\right), t=\left(t_{1}, \ldots, t_{m}\right)$ in $S$ we define $s \leq t$ if $n \leq m$ and $s_{i}=t_{i}$ for all $i=1, \ldots, n$. If $s=\left(s_{1}, \ldots, s_{k}\right) \in S$, $\sigma=\left(n_{1}, \ldots, n_{k}, \ldots\right) \in \Sigma$ and $m \in \mathbb{N}$, then we write: (i) $s<\sigma$ if $s_{i}=n_{i}$ for all $i=1, \ldots, k$, (ii) $\sigma \mid m$ for the finite sequence $\left(n_{1}, \ldots, n_{m}\right)$. For every $s \in S$ we set $I(s)=\{\sigma \in \Sigma: s<\sigma\}$; it is easy to see that the countable family $\{I(s): s \in S\}$ is a basis for $\Sigma$, consisting of open and closed sets.

For a Hausdorff topological space $X, \mathcal{K}(X)$ is the set of compact nonempty subsets of $X$. If $Y$ is a topological space, a map $F: Y \rightarrow \mathcal{K}(X)$ is said to be upper semicontinuous (usco) if for every $y \in Y$ and every open subset 
$V$ of $X$ with $F(y) \subseteq V$ there exists a neighbourhood $W$ of $y$ such that $F(W)=\bigcup\{F(t): t \in W\} \subseteq V$.

We use [F\&al] as a basic reference for the theory, notation and terminology of Banach spaces, and [J-R] for the theory of $\mathcal{K}$-analytic and countably determined topological spaces. If $X$ is a (real) Banach space, then $B_{X}$ and $B_{X^{*}}$ are the closed unit balls of $X$ and its dual $X^{*}$ respectively.

3. Strongly countably determined topological spaces. The following result is similar to Prop. 1.1 of [T] (see also Prop. 7.1.1 of [F] and [J-R]); we give a sketch of its proof.

Proposition 3.1. Let $X$ be a subspace of a compact Hausdorff space $K$. Then the following are equivalent:

(i) There exist compact subsets $K_{n}, n \in \mathbb{N}$, of $K$ such that for every compact subset $L$ of $X$ and $x \in K \backslash X$ there exists $n \in \mathbb{N}$ with $L \subseteq K_{n}$ and $x \notin K_{n}$.

(ii) There exist compact sets $B_{s}, s \in S$, in $K$ and a subset $\Sigma^{\prime}$ of $\Sigma$ such that for every compact subset $L$ of $X$ there exists $\sigma \in \Sigma^{\prime}$ with $L \subseteq \bigcap_{n=1}^{\infty} B_{\sigma \mid n} \subseteq X$ and $X=\bigcup_{\sigma \in \Sigma^{\prime}} \bigcap_{n=1}^{\infty} B_{\sigma \mid n}$.

(iii) There exists a subset $\Sigma^{\prime}$ of $\Sigma$ and an upper semicontinuous map $F: \Sigma^{\prime} \rightarrow \mathcal{K}(X)$ such that for every compact subset $L$ of $X$ there exists $\sigma \in \Sigma^{\prime}$ with $L \subseteq F(\sigma)$. In particular $X=F\left(\Sigma^{\prime}\right)$.

Proof. (i) $\Rightarrow$ (ii). For $s=\left(n_{1}, \ldots, n_{m}\right) \in S$ we put $B_{s}=\bigcap_{i=1}^{m} K_{n_{i}}$. Then we define $\Sigma^{\prime}=\left\{\sigma \in \Sigma: \emptyset \neq \bigcap_{n=1}^{\infty} B_{\sigma \mid n} \subseteq X\right\}$. Let $L$ be any nonempty compact subset of $X$. Set $M=\left\{n \in \mathbb{N}: L \subseteq K_{n}\right\}$. It is obvious that $L \subseteq$ $\bigcap_{n \in M} K_{n}$. We claim that $\bigcap_{n \in M} K_{n} \subseteq X$. Indeed, assume that there exists $u \in \bigcap_{n \in M} K_{n}$ with $u \notin X$; then by our assumption there exists $n_{0} \in M$ such that $L \subseteq K_{n_{0}}$ and $u \notin K_{n_{0}}$, a contradiction. Let $\sigma=\left(n_{1}, n_{2}, \ldots\right)$, where $\left\{n_{1}, n_{2}, \ldots\right\}$ is an enumeration of $M$; then $L \subseteq \bigcap_{n=1}^{\infty} B_{\sigma \mid n}$ and assertion (ii) is satisfied.

(ii) $\Rightarrow$ (iii). Assume without loss of generality that $B_{\sigma}=\bigcap_{n=1}^{\infty} B_{\sigma \mid n} \neq \emptyset$ for all $\sigma \in \Sigma^{\prime}$. We define a map $F: \Sigma^{\prime} \rightarrow \mathcal{K}(X)$ by $F(\sigma)=B_{\sigma}$. It is clear that it remains to prove (only) that $F$ is an upper semicontinuous map. Consider $\sigma \in \Sigma^{\prime}$ and an open set $U$ of $X$ such that $F(\sigma) \subseteq U$, that is, $F(\sigma)=\bigcap_{n=1}^{\infty} B_{\sigma \mid n} \subseteq U$. As $K$ is compact, we get $m \in \mathbb{N}$ such that $\bigcap_{n=1}^{m} B_{\sigma \mid n} \subseteq U$, hence $F\left(I(\sigma \mid m) \cap \Sigma^{\prime}\right) \subseteq U$, so $F$ is usco.

(iii) $\Rightarrow$ (ii). For each $s \in S$, let $B_{s}$ be the closure of the set $F\left(I(s) \cap \Sigma^{\prime}\right)$ in $K$. For each $\sigma \in \Sigma^{\prime}$ we have $F(\sigma) \subseteq B_{\sigma \mid n}$ for all $n \in \mathbb{N}$, so $F(\sigma) \subseteq$ $\bigcap_{n=1}^{\infty} B_{\sigma \mid n}$. If $L$ is a compact subset of $X$, then there exists $\sigma \in \Sigma^{\prime}$ such that $L \subseteq F(\sigma)$, hence $L \subseteq \bigcap_{n=1}^{\infty} B_{\sigma \mid n}$. It remains to show that $\bigcap_{n=1}^{\infty} B_{\sigma \mid n} \subseteq X$, which is true as the map $F$ is usco and $F(\sigma)=\bigcap_{n=1}^{\infty} B_{\sigma \mid n}$. 
(ii) $\Rightarrow$ (i). The set $S$ is countable. Let $\left\{s_{n}: n \in \mathbb{N}\right\}$ be an enumeration of $S$. It is enough to put $K_{n}=B_{s_{n}}$ for $n \in \mathbb{N}$.

REMARK 3.1. As is well known, the above proposition holds true if instead of pairs $(L, x)$, with $L$ a compact subset of $X$ and $x \in K \backslash X$, in (i), we consider pairs $(u, x)$ with $u \in X$ and $x \in K \backslash X$ (and with analogous modifications in (ii) and (iii)). In that case we get the well known concept of countably determined topological space (see Prop. 1.1 of [T]).

Since by assertion (iii) of the above proposition, the space $K$ can be replaced by any other compact superspace of $X$, we can give the following definition.

Definition 3.1. A topological space $X$ is called strongly countably determined $(S C D)$ if there exists a compact space $K$ such that $X \subseteq K$ and the assertions of Prop. 3.1 are satisfied.

REMARK 3.2. We note that if assertion (ii) or (iii) in the previous proposition is satisfied by $\Sigma$ instead of $\Sigma^{\prime}$ then we get the concept of a strongly $\mathcal{K}$-analytic topological (SKA) space introduced in [M-S, Def. 1.11]. It is obvious that each strongly $\mathcal{K}$-analytic space is strongly countably determined.

Examples of strongly countably determined spaces. (i) Every separable metric space $X$ is SCD. Indeed, let $K$ be a metrizable compactification of $X$. We consider a basis $\mathcal{B}=\left\{U_{n}: n \geq 1\right\}$ for the topology of $K$ that is closed for finite unions. Set $K_{n}=\bar{U}_{n} \subseteq K$ for all $n \in \mathbb{N}$. We claim that for every compact subset $L$ of $X$ and every $u \in K \backslash X$ there exists $n_{0} \in \mathbb{N}$ such that $L \subseteq K_{n_{0}}$ and $u \notin K_{n_{0}}$. So let $V$ be an open subset of $K$ such that $L \subseteq V$ and $u \notin \bar{V}$. Then for some $M \subseteq \mathbb{N}$ we have $V=\bigcup_{n \in M} U_{n}$ and by the compactness of $L$ there exists a finite subset $F$ of $M$ such that $L \subseteq \bigcup_{n \in F} U_{n} \subseteq V$. The set $\bigcup_{n \in F} U_{n}$ is also a member of $\mathcal{B}$, say $U_{n_{0}}$, thus we have $L \subseteq U_{n_{0}} \subseteq K_{n_{0}} \subseteq \bar{V}$ and of course $u \notin K_{n_{0}}$. It follows from assertion (i) of Prop. 3.1 that $X$ is SCD.

A shorter proof of this example using assertion (iii) of Prop. 3.1 goes as follows. The space $\mathcal{K}(X)$ endowed with the Hausdorff metric (which gives rise to the Vietoris topology of $\mathcal{K}(X))$ is separable and hence it is a continuous image of a subset $\Sigma^{\prime}$ of $\Sigma$.

(ii) Let $M$ be a separable metric space and $\Omega$ be a compact space. Then the space $M \times \Omega$ is SCD. This is easily verified by using the previous example. It follows immediately from Prop. 3.3 below that every closed subset $C$ of $M \times \Omega$ is SCD. If $M$ is Polish, then $C$ is SKA (see Remark 1.11.1 of [M-S]).

A continuous map $f$ from a topological space $X$ into a topological space $Y$ is said to be compact covering (see [E, p. 423] and [S-W]) if for every compact subset $L$ of $Y$ there exists a compact subset $K$ of $X$ such that $f(K)=L$. (It is clear that a compact covering map is surjective.) Obvious 
examples of compact covering maps are both projections $\pi_{1}: X \times Y \rightarrow X$ and $\pi_{2}: X \times Y \rightarrow Y$, where $X, Y$ are topological spaces. Using the concept of the compact covering map we can give still another characterization of strongly countably determined and strongly $\mathcal{K}$-analytic spaces.

Proposition 3.2. Let $X$ be a subspace of the compact space $K$. Then the following are equivalent:

(i) $X$ is $S C D$ (resp. SKA).

(ii) There exist a separable metric space (resp. a Polish space) $M, a$ closed subset $C$ of $M \times K$ and a compact covering map $f$ from $C$ onto $X$.

Proof. We examine for example the countably determined case.

(i) $\Rightarrow$ (ii). Let $F: \Sigma^{\prime} \rightarrow \mathcal{K}(X)$ be an usco map satisfying assertion (iii) of Prop. 3.1. Let $C=\bigcup\left\{\{\sigma\} \times F(\sigma): \sigma \in \Sigma^{\prime}\right\}$ be the graph of $F$. As $F$ is usco, $C$ is a closed subset of $\Sigma^{\prime} \times K$. It is immediate that the projection of $C$ onto $X$ is a compact covering map.

(ii) $\Rightarrow\left(\right.$ i). Since $C$ is a SCD space, there exists an usco map $\Phi: \Sigma^{\prime} \rightarrow$ $\mathcal{K}(C)$ satisfying assertion (iii) of Prop. 3.1. It is then easy to see that the map $F: \Sigma^{\prime} \rightarrow \mathcal{K}(X)$ defined by $F(\sigma)=f(\Phi(\sigma))$ makes $X$ a SCD space.

REMARK 3.3. (1) It should be clear after all that we may extend Def. 3.1 to obtain a wider class of Hausdorff topological spaces. So we shall call a Hausdorff space $X$ strongly countably determined (resp. strongly $\mathcal{K}$-analytic) if there exists a closed subset $C$ of a product $M \times K$, where $M$ is a separable metric (resp. Polish) space and $K$ is a compact space, and there is a compact covering map from $C$ onto $X$. It then follows as in the proof of Prop. 3.2 that there exists an usco map $F: \Sigma^{\prime} \rightarrow \mathcal{K}(X)$ such that for each compact subset $L$ of $X$ there exists $\sigma \in \Sigma^{\prime}$ with $L \subseteq F(\sigma)$. So if $X$ is a subspace of a compact space, then it is strongly countably determined (resp. strongly $\mathcal{K}$-analytic) according to Def. 3.1 and the new definition is a consistent extension of the old one.

(2) We notice that if the topological space $X$ is SCD, then there exists a separable metric space $M$ and a family $\left\{M_{K}: K \in \mathcal{K}(M)\right\}$ of compact subsets of $X$ such that: (a) if $K_{1}, K_{2} \in \mathcal{K}(M)$ with $K_{1} \subseteq K_{2}$, then $M_{K_{1}} \subseteq M_{K_{2}}$, (b) for each compact subset $L$ of $X$ there exists $K \in \mathcal{K}(M)$ such that $L \subseteq M_{K}$.

Indeed, let $\Sigma^{\prime} \subseteq \Sigma$ and $F: \Sigma^{\prime} \rightarrow \mathcal{K}(X)$ be an usco map satisfying assertion (iii) of Prop. 3.1. Set $M=\Sigma^{\prime}$ and $M_{K}=F(K)=\bigcup_{\sigma \in K} F(\sigma)$ for each $K \in \mathcal{K}(M)$. Since $F$ is usco, the set $M_{K}=F(K)$ is compact and it is easy to see that the family $\left\{M_{K}: K \in \mathcal{K}(M)\right\}$ has the desired properties. (If $X$ is SKA then we may replace $M$ by the Baire space $\Sigma$ ). Concerning the converse direction, let $X$ be a (regular) space admitting a family $\left\{M_{K}: K \in\right.$ 
$\mathcal{K}(M)\}$ of compact sets satisfying (a) and (b). If additionally $X$ is Lindelöf (or Dieudonné complete, or the relatively countably compact subsets of $X$ are relatively compact), then by Th. 4 of [C-O], $X$ is countably determined. Furthermore the method of the proof of this result and property (b) imply that $X$ is SCD (if $M$ is Polish, then $X$ is strongly $\mathcal{K}$-analytic).

In what follows, by "topological space" we mean a Hausdorff and completely regular space.

The following results give some elementary stability properties of SCD topological spaces (see also Prop. 1.13 in [M-S]).

Proposition 3.3.

(i) If $X$ is a SCD topological space and $Y$ is a closed subspace of $X$, then $Y$ is $S C D$.

(ii) If $\left(X_{n}\right)$ is any sequence of SCD topological spaces, then the space $X=\prod_{n=1}^{\infty} X_{n}$ is $S C D$.

Proof. (i) We shall use assertion (ii) of Prop. 3.1. Let $K$ be a compact superspace of $X$ and $\left\{B_{s}: s \in S\right\}$ a family of compact subsets of $K$ satisfying assertion (ii) of Prop. 3.1. Let $Z$ be a closed and hence compact subspace of $K$ such that $Y=X \cap Z$. Set $A_{s}=B_{s} \cap Z$ for all $s \in S$. Clearly $A_{s}$ is a compact set in $K$ and thus in $Z$. Let $L$ be a compact subset of $Y$. Then $L$ is a compact subset of $X$, thus there exists $\sigma \in \Sigma^{\prime}$ such that $L \subseteq \bigcap_{n=1}^{\infty} B_{\sigma \mid n} \subseteq X$, hence $L \subseteq \bigcap_{n=1}^{\infty} B_{\sigma \mid n} \cap Z \subseteq X \cap Z=Y$. Set $\Sigma^{\prime \prime}=\left\{\sigma \in \Sigma^{\prime}: \bigcap_{n=1}^{\infty} B_{\sigma \mid n} \cap Z \neq \emptyset\right\}$. Then clearly $Y=\bigcup_{\sigma \in \Sigma^{\prime \prime}} \bigcap_{n=1}^{\infty} A_{\sigma \mid n}$ and for each compact subset $L$ of $Y$ there exists $\sigma \in \Sigma^{\prime \prime}$ such that $L \subseteq \bigcap_{n=1}^{\infty} A_{\sigma \mid n}$. So the space $Y$ is strongly countably determined.

(ii) We shall use assertion (i) of Prop. 3.1 to check that $\prod_{n=1}^{\infty} X_{n}$ is strongly countably determined. For each $n \in \mathbb{N}$ consider a compact superspace $\Omega_{n}$ of $X_{n}$. It then follows, from assertion (i), that for each $n \in \mathbb{N}$ there exists a sequence $\left(K_{n}^{m}\right)_{m}$ of compact subsets of $\Omega_{n}$ such that for each compact subset $L$ of $X_{n}$ and $w \in \Omega_{n} \backslash X_{n}$ there exists $m \in \mathbb{N}$ with $L \subseteq K_{n}^{m}$ and $w \notin K_{n}^{m}$. Set $\Omega=\prod_{n=1}^{\infty} \Omega_{n}$ and $Z_{n}^{m}=\pi_{n}^{-1}\left(K_{n}^{m}\right)$ for all $n, m \in \mathbb{N}$, where $\pi_{n}$ is the $n$th projection of the space $\Omega$. We shall show that the sequence $Z_{n}^{m}, n, m \in \mathbb{N}$, separates compact subsets of $X$ from points of $\Omega \backslash X$. Let $Z$ be a compact subset of $X$ and $w=\left(w_{n}\right) \in \Omega \backslash X$. Then there exists $n \in \mathbb{N}$ with $w_{n} \notin X_{n}$, so for the compact subset $Z_{n}=\pi_{n}(Z)$ of $X_{n}$ there exists $m \in \mathbb{N}$ such that $Z_{n} \subseteq K_{n}^{m}$ and $w_{n} \notin K_{n}^{m}$. It then follows that $Z \subseteq Z_{n}^{m}$ and $w \notin Z_{n}^{m}$ and the proof is complete.

It is not true in general that a continuous image of a SCD space is a SCD space. This is because every countably determined space is a continuous image of a closed subset of a product $M \times K$, where $M$ is a separable and $K$ is a compact space, but as Example 3.1 shows, a countably determined (even 
$\mathcal{K}$-analytic) space is not necessarily SCD (see also Remark 4.3). However, the following holds.

Proposition 3.4. Let $X, Y$ be topological spaces and $f: X \rightarrow Y a$ compact covering map. If $X$ is SCD (resp. SKA), then so is $Y$.

Proof. This proof is identical to the proof of (ii) $\Rightarrow$ (i) of Prop. 3.2, so it is omitted.

COROllary 3.1. Let $(X, \tau)$ be a topological space and let d be a metric on $X$ with the following properties: (i) $(X, d)$ is a separable metric space, (ii) the metric topology $\tau_{d}$ is finer than $\tau$, (iii) a subset $K$ of $X$ is $\tau$-compact if and only if it is $\tau_{d}$-compact. Then each subspace $Y$ of $X$ endowed with the relative topology $\tau_{Y}$ is $S C D$.

Proof. It is immediate from the previous proposition.

EXAMPLE 3.1. A $\sigma$-compact space is not necessarily SCD. Let $\Gamma$ be a set with $|\Gamma|=c$ (the cardinality of the continuum) and $X=[\Gamma]^{<\omega}$ (the set of finite subsets of $\Gamma$ ), considered as a subspace of the compact space $\{0,1\}^{\Gamma}$. The space $X$ is $\sigma$-compact (in particular $\mathcal{K}$-analytic) since it can be written as $X=\bigcup_{n=1}^{\infty}[\Gamma] \leq n$, where $[\Gamma]^{\leq n}$ is the set of subsets $A$ of $\Gamma$ with $|A| \leq n$. We are going to show that $X$ is not SCD, using a result of Hájek, Lancien and Montesinos: Given an infinite cardinal $\tau$, for every ordinal $\alpha<\tau^{+}$there exists a strong Eberlein compact subset $K$ of $c_{0}(\tau)$ with height $\eta(K) \geq \alpha$. (See [H-L-M] or Lemma 2.53, pp. 71-72 in [H\&al]). Recall that a strong Eberlein compact is a compact subset of the space $[\Gamma]^{<\omega}$, where $\Gamma$ is any nonempty set.

Assume towards a contradiction that $X$ is SCD. According to (the first part of) Remark 3.3(2) there exist a separable metric space $M$ and a family $\left\{X_{K}: K \in \mathcal{K}(M)\right\}$ of compact subsets of $X$ such that (a) if $K_{1}, K_{2} \in \mathcal{K}(M)$ with $K_{1} \subseteq K_{2}$ then $X_{K_{1}} \subseteq X_{K_{2}}$, (b) for every compact subset $\Omega$ of $X$ there exists $K \in \mathcal{K}(M)$ such that $\Omega \subseteq X_{K}$. Note that every compact subset $K$ of $X$ is a strong Eberlein compact and since $|\Gamma|=c$ we have $|\eta(K)|<c^{+}$. We now consider the sets $X_{K}, K \in \mathcal{K}(M)$, as disjointly supported by the sets $\Gamma_{K}=\bigcup\left\{A \subseteq \Gamma: \mathcal{X}_{A} \in X_{K}\right\} \times\{K\}$ and set $\Delta=\bigcup\left\{\Gamma_{K}: K \in \mathcal{K}(M)\right\} ;$ since $\left|\Gamma_{K}\right| \leq c$ for all $K \in \mathcal{K}(M)$ and $|\mathcal{K}(M)| \leq c$ we get $|\Delta|=c$. Now we define a compact subset of $\{0,1\}^{\Delta}$ by setting $K_{0}=\left\{\mathcal{X}_{A}: A \subseteq \Delta\right.$ and $|A \backslash B| \leq 1$ for some $\left.B \subseteq A, \mathcal{X}_{B} \in X_{K}, K \in \mathcal{K}(M)\right\}$. It is easy to check that $K_{0}$ is a strong Eberlein compact (that is a compact subset of $[\Delta]^{<\omega}$ ). It clearly follows from condition (b) that every compact subset of $X$ is embedded in $K_{0}$, therefore $\eta(\Omega) \leq \eta\left(K_{0}\right)$ for every compact subset $\Omega$ of $X$; but then the aforementioned result from [H-L-M] implies that $\eta\left(K_{0}\right)=c^{+}$, a contradiction.

Note that Example 3.1 can also be proved using the methods of $\mathrm{A}-\mathrm{B}$. 
The following result generalizes Theorem 1.12 of $[\mathrm{M}-\mathrm{S}$. We recall that if $X$ is any Hausdorff space then the Vietoris topology $\tau_{\nu}$ on $\mathcal{K}(X)$ has a basis consisting of the subsets of $\mathcal{K}(X)$ of the form

$$
\beta\left(V_{1}, \ldots, V_{n}\right)=\left\{K \in \mathcal{K}(X): K \subseteq \bigcup_{i=1}^{n} V_{i} \text { and } K \cap V_{i} \neq \emptyset \text { for } i=1, \ldots, n\right\}
$$

where $n \in \mathbb{N}$ and $V_{1}, \ldots, V_{n}$ are open nonempty subsets of $X$ (see $[\mathrm{E}]$ ).

THEOREM 3.1. Let $X$ be a (Hausdorff and completely regular) topological space. Then the following are equivalent:

(i) $X$ is $S C D$.

(ii) $\left(\mathcal{K}(X), \tau_{\nu}\right)$ is $S C D$.

(iii) $\left(\mathcal{K}(X), \tau_{\nu}\right)$ is $C D$.

Proof. (i) $\Rightarrow$ (ii). Let $\Omega$ be a compact space with $X \subseteq \Omega$. There exists a sequence $\left(A_{n}\right)_{n}$ of compact subsets of $\Omega$ such that for each compact subset $K$ of $X$ and $w \in \Omega \backslash X$ there exists $n \in \mathbb{N}$ with $K \subseteq A_{n}$ and $w \notin A_{n}$. Put $B_{n}=\mathcal{K}\left(A_{n}\right)$ for all $n \in \mathbb{N}$. Then the space $\mathcal{K}(\Omega)$ endowed with the Vietoris topology is a compact topological space and $\left(B_{n}\right)$ is a sequence of compact subsets of $\mathcal{K}(\Omega)$. Let $K$ be a compact subset of $\mathcal{K}(X)$ and $L \in \mathcal{K}(\Omega) \backslash \mathcal{K}(X)$. Put $X_{K}=\bigcup\{A: A \in K\}$. Then $X_{K}$ is a compact subset of $X$ and $L$ is not a subset of $X$, hence there exists $w \in L$ with $w \notin X$. Then there exists $n \in \mathbb{N}$ such that $X_{K} \subseteq A_{n}$ and $w \notin A_{n}$.

We now claim that $K \subseteq B_{n}$ and $L \notin B_{n}$. Indeed, $X_{K} \subseteq A_{n}$, hence for each $A \in K$ we have $A \subseteq X_{K} \subseteq A_{n}$, that is, $A \in B_{n}=\mathcal{K}\left(A_{n}\right)$, which means that $K \subseteq B_{n}$. If we had $L \in B_{n}$, then $L \subseteq A_{n}$, hence $w \in A_{n}$, a contradiction.

(ii) $\Rightarrow$ (iii) is obvious.

(iii) $\Rightarrow$ (i). There exists a subset $\Sigma^{\prime}$ of $\Sigma$ and an usco map $\Phi: \Sigma^{\prime} \rightarrow$ $\mathcal{K}(\mathcal{K}(X))$ with $\mathcal{K}(X)=\Phi\left(\Sigma^{\prime}\right)=\bigcup_{\sigma \in \Sigma^{\prime}} \Phi(\sigma)$. Define a map $F: \Sigma^{\prime} \rightarrow \mathcal{K}(X)$ by $F(\sigma)=\bigcup \Phi(\sigma)=\bigcup\{K: K \in \Phi(\sigma)\}$. Let $L$ be a compact subset of $X$. Then $L \in \mathcal{K}(X)$, hence there exists $\sigma \in \Sigma^{\prime}$ with $L \in \Phi(\sigma)$, so $L \subseteq F(\sigma)$. It remains to prove that $F$ is upper semicontinuous. Let $F(\sigma) \subseteq V$ for some $\sigma \in \Sigma^{\prime}$ and $V$ an open subset of $X$. Consider the set $\beta(V)=\{K: K \in \mathcal{K}(X)$ and $K \subseteq V\}$, which is open in the Vietoris topology of $\mathcal{K}(X)$. Clearly $\Phi(\sigma) \subseteq \beta(V)$. By the semicontinuity of $\Phi$ there exists $n \in \mathbb{N}$ such that $\Phi\left(I(\sigma \mid n) \cap \Sigma^{\prime}\right) \subseteq \beta(V)$, that is, for all $\tau \in \Sigma^{\prime}$ with $\tau|n=\sigma| n$ we have $\Phi(\tau) \subseteq \beta(V)$. Then $K \subseteq V$ whenever $K \in \Phi(\tau)$, hence $F(\tau) \subseteq V$, and this completes the proof.

4. Strongly weakly countably determined Banach spaces. In this section we introduce and study the concept of a strongly weakly countably 
determined Banach space. The class of strongly weakly countably determined Banach spaces is a subclass of weakly countably determined (WCD) Banach spaces, for which we refer to $[\mathrm{T}]$ and $[\mathrm{F}]$.

Definition 4.1. A Banach space $X$ is called strongly weakly countably determined $(S W C D)$ if it is SCD in its weak topology.

We note that if $(X, w)$ is strongly $\mathcal{K}$-analytic then the Banach space $X$ is called strongly weakly $\mathcal{K}$-analytic (SWKA). The class of SWKA Banach spaces has been introduced in [M-S].

REMARK 4.1. (1) A Banach space $X$ is SWCD if and only if the topological space $\left(B_{X}, w\right)$ is SCD. One implication is clear because $\left(B_{X}, w\right)$ is a closed subspace of $X$. For the other, if there exists a subset $\Sigma^{\prime}$ of $\Sigma$ and an upper semicontinuous map $F: \Sigma^{\prime} \rightarrow \mathcal{K}\left(B_{X}\right)$ satisfying condition (iii) of Prop. 3.1, then $X$ is SWCD. To see this, define $\Phi: \Sigma^{\prime} \times \mathbb{N} \rightarrow \mathcal{K}(X)$ by $\Phi(\sigma, n)=n F(\sigma)$, which satisfies condition (iii) of Prop. 3.1 and makes $X$ a SWCD space.

(2) It is easy to see that a Banach space $X$ is SWCD if and only if there exists a sequence $\left(K_{n}\right)$ of $w^{*}$-compact subsets of $X^{* *}$ with the following property: For each weakly compact subset $L$ of $X$ and each $x^{* *} \in X^{* *} \backslash X$ there exists $n \in \mathbb{N}$ such that $L \subseteq K_{n}$ and $x^{* *} \notin K_{n}$. Indeed, if $X$ is SWCD, then the ball $B_{X}$ of $X$ endowed with the weak topology is SCD, as a closed subspace. So there exists a sequence $\left(K_{n}\right)$ of $w^{*}$-compact subsets of $B_{X^{* *}}$ which satisfies condition (i) of Prop. 3.1. It is easy to see that the countable family $m K_{n}, m, n \geq 1$, has the desired properties. Conversely, if there exists such a sequence $\left(K_{n}\right)$, then the sequence $\left(K_{n} \cap B_{X^{* *}}\right)$ of $w^{*}$-compact subsets of $B_{X^{* *}}$ satisfies assertion (i) of Prop. 3.1, so $\left(B_{X}, w\right)$ is strongly countably determined, hence $X$ is SWCD.

(3) It follows from Prop. 3.3 that the class of SWCD Banach spaces is stable under closed subspaces and finite products. From Th. 3.1 we find that a Banach space $X$ is SWCD if and only if the space $\mathcal{K}(X)$ with the Vietoris topology (induced by the weak topology of $X$ ) is countably determined.

Examples of SWCD Banach spaces. (i) Every SWKA Banach space is SWCD. (Thus in particular every SWCG Banach space is SWCD; see (M-S].)

(ii) Every separable Banach space $X$ with separable dual is SWCD. This is because the unit ball $\left(B_{X}, w\right)$ is separable and metrizable. In particular $c_{0}(\mathbb{N})$ is SWCD but not SWKA. (See Prop. 1.9 and Cor. 1.10 of [M-S].)

(iii) Every separable Banach space $X$ with the Schur property is SWCD. Actually, Cor. 3.1 implies that every subset $A$ of $X$ with the weak topology is a $\mathrm{SCD}$ topological space. Recall that $\ell^{1}(\mathbb{N})$ has the Schur property. 
(iv) If $X^{*}$ is separable, $Y$ reflexive, and $Z$ a closed subspace of the Banach space $X \times Y$, then $Z$ is SWCD (see Remark 4.1(3)).

The following is a useful characterization of SWCD spaces (cf. Prop. 1.7 of [M-S] for a similar characterization of SWKA Banach spaces). Its analogue for WCD spaces, first proved in [M1 (see also Th. 3.16 in A-M]) and independently in [C-O, Cor. 4.1], states that a Banach space $X$ is WCD if and only if there exists a separable metric space $M$ and a family $\left\{W_{K}\right.$ : $K \in \mathcal{K}(M)\}$ of weakly compact subsets of $X$ such that (a) $W_{K_{1}} \subseteq W_{K_{2}}$ if $K_{1} \subseteq K_{2}$, (b) $\bigcup\left\{W_{K}: K \in \mathcal{K}(M)\right\}$ is a total subset of $X$. (The analogous characterization of weakly $\mathcal{K}$-analytic Banach spaces is due to Talagrand [T, Prop. 6.13].

Proposition 4.1. Let $X$ be a Banach space. Then the following are equivalent:

(i) $X$ is $S W C D$.

(ii) There exists a separable metric space $M$ and a family $\left\{W_{K}: K \in\right.$ $\mathcal{K}(M)\}$ of weakly compact subsets of $X$ (resp. of $B_{X}$ ) such that: (a) if $K_{1}, K_{2} \in \mathcal{K}(M)$ with $K_{1} \subseteq K_{2}$, then $W_{K_{1}} \subseteq W_{K_{2}}$, (b) for every weakly compact subset $L$ of $X$ (resp. of $B_{X}$ ) there exists $K \in$ $\mathcal{K}(M)$ such that $L \subseteq W_{K}$.

Proof. Since a Banach space $X$ endowed with the weak topology is Hausdorff and completely regular, and furthermore relatively countably compact sets of $(X, w)$ are relatively compact, the result follows immediately from Remark 3.3 . However, we prefer to give a more direct proof. $3.3(2)$

(i) $\Rightarrow$ (ii). It is an immediate consequence of the easy part of Remark

(ii) $\Rightarrow$ (i). Let $\left\{W_{K}: K \in \mathcal{K}(M)\right\}$ be a family of weakly compact subsets of $B_{X}$ satisfying conditions (a) and (b) of assertion (ii). It then follows from the characterization of WCD Banach spaces, mentioned above, that $X$ is WCD, hence $\left(B_{X^{*}}, w^{*}\right)$ is a Gul'ko compact. (In particular this space is angelic.) Let $\left\{V_{n}: n \geq 1\right\}$ be a countable basis for the topology of the separable metric space $\mathcal{K}(M)$. For each $n \in \mathbb{N}$ define $\Omega_{n}$ to be the $w^{*}$ closure of the set $\bigcup\left\{W_{K}: K \in V_{n}\right\}$ in $B_{X^{* *}}$. It is then easy to verify that the sequence $\left(\Omega_{n}\right)$ of $w^{*}$-compact subsets of $B_{X^{* *}}$ satisfies condition (i) of Prop. 3.1

The class of SWCD Banach spaces is not stable under continuous linear maps (cf. Remark 1.16 in [M-S]). Indeed, every separable Banach space is a quotient of $\ell^{1}(\mathbb{N})$, but as can be proved below (see Remark 4.3), no separable Banach space not containing $\ell^{1}(\mathbb{N})$ with nonseparable dual is SWCD. On the other hand, we have the following positive result. 
Proposition 4.2. Let $X$ be a Banach space and $Y$ a reflexive subspace of $X$. Then $X$ is $S W C D$ (resp. $S W K A$ ) if and only if $X / Y$ is $S W C D$ (resp. $S W K A)$.

Proof. As proved in [S-W, Th. 2.7], if $Y$ is a reflexive subspace of a Banach space $X$, then the quotient map $q: X \rightarrow X / Y$ is a weakly compact covering (in the sense of Section 1). Actually, it is rather easy to see that if $K$ is a weakly compact subset of $X / Y$ and $\delta>0$, then $\Omega=B_{X}(0, \delta) \cap q^{-1}(K)$ is weakly compact in $X$ and if in addition $\delta>\sup \{\|\hat{x}\|: \hat{x} \in K\}$, then $q(\Omega)=K$. Let us examine the SWCD case (the SWKA case uses Prop. 1.7 of $[\mathrm{M}-\mathrm{S}]$ ).

One implication is an easy consequence of Prop. 3.4. For the other, let $\left\{W_{K}: K \in \mathcal{K}(M)\right\}$ be a family of weakly compact subsets of $B_{X / Y}$ making $X / Y$ a SWCD space according to Prop. 4.1. Set $\Omega_{K}=B_{X} \cap q^{-1}\left(W_{K}\right)$ for each $K \in \mathcal{K}(M)$. Then each $\Omega_{K}$ is a weakly compact subset of $B_{X}$ and if $K_{1} \subseteq K_{2}$, then $\Omega_{K_{1}} \subseteq \Omega_{K_{2}}$. Let $\Omega$ be a weakly compact subset of $B_{X}$. Then $q(\Omega) \subseteq q\left(B_{X}\right) \subseteq B_{X / Y}$, thus there exists some $K$ such that $q(\Omega) \subseteq W_{K}$; so $\Omega \subseteq q^{-1}\left(W_{K}\right) \cap B_{X}=\Omega_{K}$, which completes the proof.

Remark 4.2. The analogue of Prop. 4.2 for WCD and weakly $\mathcal{K}$-analytic Banach spaces is also true (see also Th. 2.7 of $[\mathrm{S}-\mathrm{W}]$ ). We use the characterization of WCD spaces mentioned above and the corresponding characterization of weakly $\mathcal{K}$-analytic Banach spaces of Talagrand ([T, Prop. 6.13]).

Lemma 4.1. Let $\Gamma$ be a nonempty set, $M$ a separable metric space and $\left\{\Gamma_{K}: K \in \mathcal{K}(M)\right\}$ a family of finite subsets of $\Gamma$ such that: (i) $\Gamma=\bigcup\left\{\Gamma_{K}\right.$ : $K \in \mathcal{K}(M)\}$, (ii) if $K_{1}, K_{2} \in K(M)$ with $K_{1} \subseteq K_{2}$, then $\Gamma_{K_{1}} \subseteq \Gamma_{K_{2}}$, (iii) each $\Gamma_{K}$ is finite. Then the set $\Gamma$ is countable.

Proof. Assume for contradiction that $\Gamma$ is uncountable; we may assume that $|\Gamma|=\omega_{1}$ and let $\Gamma=\left\{\gamma_{\xi}: \xi<\omega_{1}\right\}$ be an enumeration of $\Gamma$. For each $\xi<\omega_{1}$ pick $K_{\xi} \in \mathcal{K}(M)$ such that $\gamma_{\xi} \in \Gamma_{K_{\xi}}$. We claim that $\left\{K_{\xi}: \xi<\omega_{1}\right\}$ is a countable subset of $\mathcal{K}(M)$. If the claim is not true, then since the space $\mathcal{K}(M)$ endowed with the Vietoris topology is separable and metrizable, there would exist a sequence $\left(K_{\xi_{n}}\right)$ of distinct sets of $\mathcal{K}(M)$ and $K \in \mathcal{K}(M)$ such that $K_{\xi_{n}} \stackrel{\tau_{\nu}}{\rightarrow} K$. It then follows that the set $W=\bigcup_{n=1}^{\infty} K_{\xi_{n}} \cup K$ is compact in $M$ and $\Gamma_{K_{\xi_{n}}} \subseteq \Gamma_{W}$ for every $n \geq 1$, so $\left\{\gamma_{\xi_{n}}: n \geq 1\right\} \subseteq \Gamma_{W}$, which is a contradiction.

For another proof of the previous lemma one can see [M2, Remark 1.4].

The following results of this section generalize [M-S, Th. 2.6, Cor. 2.7, Th. 2.8 and Example 2.9]. We omit the proofs of the next two theorems as they follow the lines of the proofs of Ths. 2.7 and 2.8 in [M-S]. The only difference is that instead of the characterization of SWKA Banach spaces 
and Lemma 2.1 of $[\mathrm{M}-\mathrm{S}$ ] we use the characterization of SWCD spaces and Lemma 4.1 of the present paper.

The first result is a characterization of separable SWCD spaces not containing $\ell^{1}(\mathbb{N})$.

TheOREM 4.1. Let $X$ be a separable Banach space not containing $\ell^{1}(\mathbb{N})$. Then $X$ is $S W C D$ if and only if $X$ has separable dual.

Corollary 4.1. Every $S W C D$ Banach space $E$ not containing $\ell^{1}(\mathbb{N})$ is Asplund and (hence) WCG.

Proof. Every separable subspace of $E$ has separable dual, so $E$ is Asplund. As $E$ is Asplund and WCD, it is WCG.

REMARK 4.3. It follows immediately from Th. 4.1 that a Banach space $X$ not containing $\ell^{1}(\mathbb{N})$ with nonseparable dual (for instance the James tree space) is not SWCD. Such a Banach space can also be used to show that the class of SCD topological spaces is not stable under continuous images; indeed, the identity map $I:(X,\|\cdot\|) \rightarrow(X, w)$ is continuous, the space $(X,\|\cdot\|)$ is Polish, hence SCD, but the space $(X, w)$ is not SCD.

In the next theorem $T$ denotes the dyadic tree, that is, $T=\bigcup_{n=1}^{\infty}\{0,1\}^{n}$ ordered (as the tree $S=\bigcup_{n=0}^{\infty} \mathbb{N}^{n}$ ) by the relation " $s$ is an initial segment of $t$ ", denoted by $s \leq t$.

TheOrem 4.2. Let $X$ be a Banach space and $\left(e_{s}\right)_{s \in T}$ be a bounded family of $X$. Assume that (i) for no chain $\left(t_{n}\right)$ of $T$ is the sequence $\left(e_{t_{n}}\right)$ weakly convergent, (ii) for every antichain $\left(s_{n}\right)$ of $T$ there exists a subsequence $\left(s_{n}^{\prime}\right)$ of $\left(s_{n}\right)$ such that the sequence $\left(e_{s_{n}^{\prime}}\right)$ is weakly convergent. Then the space $X$ is not $S W C D$.

REMARK 4.4. As an application of Th. 4.2 we can prove that a separable weakly sequentially complete Banach space with an unconditional basis need not be SWCD. Actually it is proved in [M-S, Example 2.9] that the space $X_{0}$ considered by Batt and Hiermeyer $[\mathrm{B}-\mathrm{H}]$ is not SWKA. Following the method of proof of that example, one can prove that $X_{0}$ is not even SWCD.

5. Direct sums of SWCD Banach spaces. In this section we examine direct sums of SWCD and SWKA Banach spaces. We show that in some cases the direct sum of countably many SWCD or SWKA Banach spaces is again SWCD or SWKA. But the direct sum of uncountably many SWCD spaces is not necessarily SWCD. In particular we show that $c_{0}\left(\omega_{1}\right)$ is not a SWCD space. Using this result we show that a Banach space of the form $C(K)$, where $K$ is a compact space, is SWCD if and only if $K$ is countable. 
Proposition 5.1. Let $\left(X_{n}\right)$ be a sequence of $S W C D$ (resp. SWKA) Banach spaces and $p \geq 1$. Then the space $X=\left(\sum_{n=1}^{\infty} \oplus X_{n}\right)_{p}$ is $S W C D$ (resp. $S W K A)$.

Proof. We consider each $X_{n}$ with the weak topology and let $\tau$ be the product topology on $\prod_{n=1}^{\infty} X_{n}$, that is, $\tau$ is the pointwise weak topology. Clearly the pointwise weak topology is coarser than the weak topology on $X$.

Case I. Let $p>1$. In this case the weak topology coincides with the pointwise weak topology on $B_{X}$ and the space $\left(B_{X}, w\right)$ is a closed subset of $\left(\prod_{n=1}^{\infty} X_{n}, \tau\right)$. So the desired conclusion follows immediately from Prop. 3.3 .

Case II. Let $p=1$. In this case $B_{X}$ is a closed subset of $X$ endowed with the pointwise weak topology. It is also not difficult to verify both of the following facts:

FACT I. Every compact subset of the space $\left(\prod_{n=1}^{\infty} X_{n}, \tau\right)$ is sequentially compact; actually, it is an Eberlein compact set.

FACT II. Let $\Omega$ be a relatively compact subset of $B_{X}$ in the $\tau$ topology. Then $\Omega$ is weakly relatively compact if and only if for each $\epsilon>0$ there exists $N \in \mathbb{N}$ such that $\sum_{k=N}^{\infty}\left\|x_{k}\right\|<\epsilon$ for all $x=\left(x_{k}\right) \in \Omega$.

Let us prove the SWKA case, using Prop. 1.7 of [M-S]. (The SWCD case is similar using the corresponding Prop. 4.1.) For each $k \in \mathbb{N}$ there exists a family $\left\{\Omega_{\sigma}^{k}: \sigma \in \Sigma\right\}$ of weakly compact subsets of $B_{X_{k}}$ such that:

(i) If $\sigma, \tau \in \Sigma$ with $\sigma \leq \tau$, then $\Omega_{\sigma}^{k} \subseteq \Omega_{\tau}^{k}$, where $\sigma \leq \tau$ means that $\sigma(n) \leq \tau(n)$ for all $n \in \mathbb{N}$.

(ii) For each weakly compact subset $L$ of $B_{X_{k}}$, there exists $\sigma \in \Sigma$ such that $L \subseteq \Omega_{\sigma}^{k}$.

For every pair of sequences $\left(m_{k}\right) \subseteq \mathbb{N}$ and $\left(\sigma_{k}\right) \subseteq \Sigma$ set

$$
\Omega_{\left(\left(m_{k}\right),\left(\sigma_{k}\right)\right)}=\left\{\left(x_{k}\right) \in B_{X}: x_{k} \in \Omega_{\sigma_{k}}^{k} \forall k \text { and } \sum_{k=m_{i}}^{\infty}\left\|x_{k}\right\| \leq 1 / i \forall i \geq 1\right\} \text {. }
$$

Then we have the following:

(a) Each set $\Omega_{\left(\left(m_{k}\right),\left(\sigma_{k}\right)\right)}$ is weakly compact. Indeed, let $\epsilon>0$. Then there exists $i \in \mathbb{N}$ with $1 / i<\epsilon$, so if $x=\left(x_{k}\right) \in \Omega_{\left(\left(m_{k}\right),\left(\sigma_{k}\right)\right)}$, then $\sum_{k=m_{i}}^{\infty}\left\|x_{k}\right\|<1 / i$. It easily follows that $\Omega_{\left(\left(m_{k}\right),\left(\sigma_{k}\right)\right)}$ is $\tau$-compact, hence weakly compact (Fact II).

(b) If $\left(m_{k}\right),\left(l_{k}\right) \subseteq \mathbb{N}$ and $\left(\sigma_{k}\right),\left(\tau_{k}\right) \subseteq \Sigma$ with $m_{k} \leq l_{k}$ and $\sigma_{k} \leq \tau_{k}$ for all $k \in \mathbb{N}$, then $\Omega_{\left(\left(m_{k}\right),\left(\sigma_{k}\right)\right)} \subseteq \Omega_{\left(\left(l_{k}\right),\left(\tau_{k}\right)\right)}$. Indeed, if $x=\left(x_{k}\right) \in$ $\Omega_{\left(\left(m_{k}\right),\left(\sigma_{k}\right)\right)}$, then $x_{k} \in \Omega_{\sigma_{k}}^{k}$ for all $k$ and $\sum_{k=m_{i}}^{\infty}\left\|x_{k}\right\|<1 / i$ for all $i$. It follows that $x_{k} \in \Omega_{\tau_{k}}^{k}$ for all $k$ and $\sum_{k=l_{i}}^{\infty}\left\|x_{k}\right\|<1 / i$ for all $i$, hence $x \in \Omega_{\left(\left(l_{k}\right),\left(\tau_{k}\right)\right)}$. 
(c) Let $Z$ be a weakly compact subset of $B_{X}$. For each $k$ set $Z_{k}=p_{k}(Z)$. Then $Z_{k}$ is a weakly compact subset of $B_{X_{k}}$, so there exists $\sigma_{k} \in \Sigma$ such that $Z_{k} \subseteq \Omega_{\sigma_{k}}^{k}$. Since $Z$ is weakly compact, for each $i \geq 1$ there exists $m_{i} \in \mathbb{N}$ such that $\sum_{k=m_{i}}^{\infty}\left\|x_{k}\right\|<1 / i$ for all $x=\left(x_{k}\right) \in Z$.

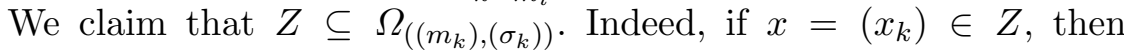
$x_{k} \in Z_{k} \subseteq \Omega_{\sigma_{k}}^{k}$ and for each $i \geq 1$ we have $\sum_{k=m_{i}}^{\infty}\left\|x_{k}\right\|<1 / i$ and the claim is proved.

Therefore the space $X$ is SWKA.

We now turn to the case $p=0$. We first observe that a direct $c_{0}$-sum $X=$ $\left(\sum_{n=1}^{\infty} \oplus X_{n}\right)_{0}$ of (nontrivial) Banach spaces is never SWKA. This is because the space $c_{0}(\mathbb{N})$ is isometrically embedded in $X$ and as we have already noticed, $c_{0}(\mathbb{N})$ is not SWKA. We do not know whether a countable $c_{0}$-sum of (separable) SWCD Banach spaces is again a SWCD space. However, the following special case can be proved.

Proposition 5.2. Let $\left(X_{n}\right)$ be a sequence of separable (SWCD) Banach spaces each of which has separable dual or has the Schur property. Then the space $X=\left(\sum_{n=1}^{\infty} \oplus X_{n}\right)_{0}$ is $S W C D$. (For instance, for each $n, X_{n}=c_{0}(\mathbb{N})$ or $X_{n}=\ell^{1}(\mathbb{N})$.)

Proof. Set $Z=\prod_{n=1}^{\infty} B_{X_{n}}$ and endow $Z$ with the pointwise weak topology $\tau$. Then $B_{X}$ is a subset of $Z$ and the weak topology on $B_{X}$ coincides with the relative topology that induces $\tau$ on $B_{X}$. By our assumption for each $n \in \mathbb{N}$ there exists a separable metrizable topology $\tau_{d_{n}}$ on $B_{X_{n}}$, finer than the weak topology and having the same compact sets. (If $X_{n}$ has separable dual then the weak topology on $B_{X}$ is metrizable, and if $X_{n}$ has the Schur property then the norm topology and the weak topology have the same compact sets.) We now consider each $B_{X_{n}}$ with the metric topology $\tau_{d_{n}}$ and give $Z$ the corresponding product topology $\tau_{d}$. Then $\left(Z, \tau_{d}\right)$ is a separable metrizable space and $\tau_{d}$ is finer than $\tau$. Let $\Omega$ be a $\tau$-compact set in $Z$ and let $x_{k}=\left(x_{n}^{k}\right)_{n=1}^{\infty}, k \geq 1$, be a sequence in $\Omega$. By the compactness of $\Omega$ each set $p_{n}(\Omega) \subseteq B_{X_{n}}$ is weakly compact (where $p_{n}$ is the projection on the $n$th coordinate). Thus for each $n \geq 1,\left(x_{n}^{k}\right)_{k}$ has a weakly convergent subsequence. By a diagonal argument it follows that $\left(x_{k}\right)_{k}$ has a convergent subsequence in $\Omega$, say $x_{k_{m}} \stackrel{\tau}{\rightarrow} x$, which means that $x_{k_{m}} \stackrel{\tau_{d}}{\rightarrow} x$. So $\Omega$ is $\tau_{d}$-compact and by Cor. 3.1 the space $\left(B_{X}, w\right)$ is SCD, that is, $X$ is SWCD.

It is natural to ask whether the uncountable direct sum $\left(\sum_{\gamma \in \Gamma} \oplus X_{\gamma}\right)_{p}$ with $p=0$ or $p>1$ of a family $\left(X_{\gamma}\right)_{\gamma \in \Gamma}$ of SWCD Banach spaces is also a SWCD Banach space. As we shall see, the answer to this question is negative.

Theorem 5.1. For each $\xi<\omega_{1}$ let $E_{\xi}$ be a Banach space having a normalized Schauder basis $\left(e_{(n, \xi)}\right)$ with no weakly convergent subsequence. 
Then the Banach space $E=\left(\sum_{\xi<\omega_{1}} \oplus E_{\xi}\right)_{p}$ where $p=0$ or $p>1$ is $W C G$, but not $S W C D$.

Proof. It is well known that a $c_{0}$ or $\ell^{p}, p>1$, direct sum of WCG Banach spaces is WCG. Let $K$ be a weakly compact subset of $E$. Then for each $\xi<\omega_{1}$ put $N_{\xi}=\left\{n \in \mathbb{N}: e_{(n, \xi)} \in K\right\}$. The set $N_{\xi}$ is finite as $K$ is weakly compact and the sequence $\left(e_{(n, \xi)}\right)$ has no weakly convergent subsequence. Define $m(K, \xi)=1$ if $N_{\xi}=\emptyset$ and $m(K, \xi)=\max N_{\xi}$ if $N_{\xi} \neq \emptyset$. Consider the map $F: \mathcal{K}(E) \rightarrow \mathcal{K}\left(\mathbb{N}^{\omega_{1}}\right)$ defined by $F(K)=\prod_{\xi<\omega_{1}}\{1, \ldots, m(K, \xi)\}$. The map $F$ has the following properties:

(i) If $K_{1}, K_{2} \in \mathcal{K}(E)$ with $K_{1} \subseteq K_{2}$, then clearly $F\left(K_{1}\right) \subseteq F\left(K_{2}\right)$.

(ii) If a family $\mathcal{C}$ of weakly compact subsets of $E$ dominates the weakly compact subsets of $E$, then the family $\{F(C): C \in \mathcal{C}\}$ dominates the compact subsets of $\mathbb{N}^{\omega_{1}}$. Indeed, if $\Omega \in \mathcal{K}\left(\mathbb{N}^{\omega_{1}}\right)$ then there exists $\sigma \in \mathbb{N}^{\omega_{1}}$ such that $\Omega \subseteq \Omega(\sigma)=\prod_{\xi<\omega_{1}}\{1, \ldots, \sigma(\xi)\}$. Define $K=\left\{e_{(n, \xi)}: n \leq\right.$ $\left.\sigma(\xi), \xi<\omega_{1}\right\} \cup\{0\}$. The set $K$ is weakly compact in $E$ and $F(K)=\Omega(\sigma)$. There exists $C \in \mathcal{C}$ such that $K \subseteq C$, hence $\Omega \subseteq \Omega(\sigma)=F(K) \subseteq F(C)$.

Assume towards a contradiction that the space $E$ is SWCD. Then, by the first part of Remark 3.3(2), there exist a separable metric space $M$ and a family $\left\{X_{K}: K \in \mathcal{K}(M)\right\}$ of weakly compact subsets of $E$ such that: (a) for all $K_{1}, K_{2} \in \mathcal{K}(M)$ with $K_{1} \subseteq K_{2}$ we have $X_{K_{1}} \subseteq X_{K_{2}}$, (b) for every weakly compact subset $L$ of $E$ there exists $K \in \mathcal{K}(M)$ such that $L \subseteq X_{K}$. It then follows that the family $\left\{F\left(X_{K}\right): K \in \mathcal{K}(M)\right\}$ dominates the compact sets of $\mathbb{N}^{\omega_{1}}$, in particular, $\mathbb{N}^{\omega_{1}}=\bigcup\left\{F\left(X_{K}\right): K \in \mathcal{K}(M)\right\}$ and $F\left(X_{K_{1}}\right) \subseteq F\left(X_{K_{2}}\right)$ whenever $K_{1}, K_{2} \in \mathcal{K}(M)$ with $K_{1} \subseteq K_{2}$. Since the space $\mathbb{N}^{\omega_{1}}$ is realcompact, Th. 4 of [C-O] (see also Remark $3.3(2)$ ) implies that $\mathbb{N}^{\omega_{1}}$ is countably determined and hence Lindelöf, a contradiction (cf. Remark 5.1 below).

REMARK 5.1. An alternative way to get a contradiction is the following: Let $D$ be a closed and discrete subset of $\mathbb{N}^{\omega_{1}}$ with cardinality $\omega_{1}$. (We thank Professor D. H. Fremlin for pointing out to us that such a subset exists.) For each $K \in \mathcal{K}(M)$ define $D_{K}=D \cap F\left(X_{K}\right)$. Then (i) $D=\bigcup\left\{D_{K}\right.$ : $K \in \mathcal{K}(M)\}$, (ii) $D_{K_{1}} \subseteq D_{K_{2}}$ whenever $K_{1}, K_{2} \in \mathcal{K}(M)$ with $K_{1} \subseteq K_{2}$, (iii) each $D_{K}$ is finite. Then Lemma 4.1 shows that the set $D$ is countable, a contradiction.

Corollary 5.1. Let $\left\{X_{\xi}: \xi<\omega_{1}\right\}$ be a family of Banach spaces and $X=\left(\sum_{\xi<\omega_{1}} \oplus X_{\xi}\right)_{p}$, where $p=0$ or $p>1$. Then:

(i) If for each $\xi<\omega_{1}, X_{\xi}=\ell^{1}(\mathbb{N})$ or $X_{\xi}=c_{0}(\mathbb{N})$, then $X$ is $W C G$, but not $S W C D$. In particular, $c_{0}\left(\omega_{1}\right)$ is not $S W C D$.

(ii) If $p=0$ (and uncountably many of $X_{\xi}$ are nontrivial), then $X$ is not $S W C D$. 
Proof. (i) The usual basis of $\ell^{1}(\mathbb{N})$ and the summing basis of $c_{0}(\mathbb{N})$ have no weakly convergent subsequence, so the previous theorem can be applied. If we set $p=0$ and $X_{\xi}=c_{0}(\mathbb{N})$ we get $X=c_{0}\left(\omega_{1}\right)$, so $c_{0}\left(\omega_{1}\right)$ is not SWCD.

(ii) Assume without loss of generality that $X_{\xi} \neq\{0\}$ for all $\xi<\omega_{1}$. Then $c_{0}\left(\omega_{1}\right)$ is embedded in $X$, hence $X$ is not SWCD.

REMARK 5.2. Since $c_{0}\left(\omega_{1}\right)$ (being WCG) is WKA, we get still another example of a $\mathcal{K}$-analytic topological space which is not SCD (cf. Example 3.1.

According to Cor. 1.10 of [M-S], if $K$ is a compact space, then the space $C(K)$ is SWKA if and only if $K$ is a finite set. In the case of SWCD Banach spaces we have the following analogue of this result.

TheOREM 5.2. Let $K$ be a compact space. Then the space $C(K)$ is $S W C D$ if and only if $K$ is countable.

Proof. If $K$ is countable, then the dual $C(K)^{*}=\ell^{1}(K)$ of $C(K)$ is separable, hence $C(K)$ is SWCD. Conversely, assume that $C(K)$ is SWCD and, towards a contradiction, that $K$ is not metrizable. It then follows that the topological weight $w(K)=\alpha$ of $K$ is uncountable. Since $K$ is a Gul'ko compact, by a result of Argyros and Negrepontis there exists a pairwise disjoint family $\left\{U_{\xi}: \xi<\alpha\right\}$ of open nonempty subsets of $K$ (see $[\mathrm{A}-\mathrm{N}$ ]). Then $c_{0}(\alpha)$ is embedded in $C(K)$, hence $c_{0}(\alpha)$ is $\mathrm{SWCD}$, a contradiction as $\alpha$ is uncountable. So $K$ is metrizable. Now if $K$ is uncountable, then $C(K)$ is isomorphic to $C[0,1]$, by Milyutin's theorem. It follows that $C(K)$ contains isomorphically any separable Banach space. In particular $C(K)$ contains a separable Banach space with nonseparable dual not containing $\ell^{1}(\mathbb{N}$ ) (for example the James tree space $J T$ ), and this contradicts Th. 4.1.

\section{Open questions}

(1) Let $X$ be a SWCD Banach space.

(a) Is then $X$ a subspace of a WCG or at least weakly $\mathcal{K}$-analytic?

(b) Assume that $X$ does not contain $\ell^{1}(\mathbb{N})$. Is then $X$ isomorphic to a closed subspace of a direct sum $Y \oplus Z$, where $Y^{*}$ is separable and $Z$ is reflexive? (cf. Cor. 4.1)

(2) Must a separable, weakly sequentially complete space which contains $\ell^{1}(\mathbb{N})$ hereditarily be SWKA or SWCD? (cf. Question (c) in $[\mathrm{S}-\mathrm{W}]$ )

\section{References}

[A-B] S. Argyros and Y. Benyamini, Universal WCG Banach spaces and universal Eberlein compacts, Israel J. Math. 38 (1987), 305-320. 
[A-M] S. Argyros and S. Mercourakis, On weakly Lindelöf Banach spaces, Rocky Mountain J. Math. 23 (1993), 395-446.

[A-N] S. Argyros and S. Negrepontis, On weakly $\mathcal{K}$-countably determined spaces of continuous functions, Proc. Amer. Math. Soc. 87 (1983), 731-736.

[B-H] J. Batt and W. Hiermeyer, On compactness in $L_{p}(\mu, X)$ in the weak topology and in the topology $\sigma\left(L_{p}(\mu, X), L_{q}\left(\mu, X^{\prime}\right)\right)$, Math. Z. 182 (1983), 409-423.

[C-O] B. Cascales and J. Orihuela, A sequential property of set-valued maps, J. Math. Anal. Appl. 156 (1991), 86-100.

[E] R. Engelking, General Topology, PWN, Warszawa, 1977.

[F] M. Fabian, Gâteaux Differentiability of Convex Functions and Topology, Wiley, New York, 1997.

[F\&al] M. Fabian, P. Habala, P. Hájek, S. V. Montesinos, J. Pelant and V. Zizler, Functional Analysis and Infinite Dimensional Geometry, CMS Books Math. 8, Springer, New York, 2001.

[H-L-M] P. Hájek, G. Lancien and V. Montesinos, Universality of Asplund spaces, Proc. Amer. Math. Soc. 135 (2007), 2031-2035.

[H\&al] P. Hájek, V. Montesinos, J. Vanderwerff and V. Zizler, Biorthogonal Systems in Banach Spaces, CMS Books Math. 26, Springer, New York, 2008.

[J-R] J. E. Jayne and C. A. Rogers, $K$-analytic sets, in: Analytic Sets, Academic Press, London, 1980, 1-181.

[M1] S. Mercourakis, Corson compact spaces and the structure of weakly $\mathcal{K}$-analytic Banach spaces, Doctoral dissertation, Athens Univ., 1983 (in Greek).

[M2] -, On weakly countably determined Banach spaces, Trans. Amer. Math. Soc. 300 (1987), 307-327.

[M-S] S. Mercourakis and E. Stamati, A new class of weakly $\mathcal{K}$-analytic Banach spaces, Comment. Math. Univ. Carolin. 47 (2006), 291-312.

[S-W] G. Schlüchtermann and R. F. Wheeler, On strongly WCG Banach spaces, Math. Z. 199 (1998), 387-398.

[T] M. Talagrand, Espaces de Banach faiblement $\mathcal{K}$-analytiques, Ann. of Math. 110 (1979), 407-438.

K. K. Kampoukos, S. K. Mercourakis

Department of Mathematics

University of Athens

Panepistemiopolis

15784 Athens, Greece

E-mail: kkamp@math.uoa.gr

smercour@math.uoa.gr

Received 14 October 2009;

in revised form 27 January 2010 